\title{
Expression of Kruppel-like factor 8 and Ki67 in lung adenocarcinoma and prognosis
}

\author{
YIFEI LIU ${ }^{1,2}$, XIUFANG YAO ${ }^{3}$, QING ZHANG $^{2}$, LI QIAN $^{2}$, JIA FENG $^{2}$, \\ TINGTING BIAN $^{2}$, JIANGUO ZHANG ${ }^{2}$ and YE TIAN $^{1}$ \\ ${ }^{1}$ Department of Radiotherapy and Oncology, The Second Affiliated Hospital of Soochow University, Suzhou, Jiangsu 215000; \\ ${ }^{2}$ Department of Pathology, Affiliated Hospital of Nantong University, Nantong, Jiangsu 226001; ${ }^{3}$ Department of Pathology, \\ The First People's Hospital of Rudong, Nantong, Jiangsu 226401, P.R. China
}

Received May 24, 2016; Accepted April 7, 2017

DOI: $10.3892 /$ etm.2017.4632

\begin{abstract}
Kruppel-like factor 8 (KLF8) belongs to the KLF family and has various roles in the regulation of the cell cycle, proliferation and tumor genesis. KLF8 is overexpressed in gastric, ovarian, breast and renal cancer. Additionally, KLF8 may affect invasion and metastasis of tumors. However, whether KLF8 also acts as an ontogeny in lung adenocarcinoma (LAC) remains unknown. The aim of the present study was to determine the association between KLF8 expression and various clinical and pathological parameters. Western blot assays and immune histochemistry analyses revealed that KLF8 level in LAC tissues was higher than that in the normal lung tissues and KLF8 expression was significantly associated with clinical variables $(\mathrm{P}<0.05)$. Kaplan-Meier curves revealed that high expression of KLF8 was related to poor prognosis in patients with LAC. The present study also demonstrated that KLF8 was involved in the progression of lung adenocarcinoma. This data suggested that KLF8 may act as a prognostic factor in lung adenocarcinoma progression.
\end{abstract}

\section{Introduction}

Lung cancer is a threat to people's health and life, and according to the pathological features it may be divided into small cell lung cancer and non-small cell lung cancer (NSCLC). NSCLC accounts for $\sim 80 \%$ of all lung cancer cases (1). Adenocarcinoma is the most common pathological type of NSCLC (1). Although surgery, comprehensive treatment and postoperative chemotherapy have been applied to

Correspondence to: Professor Ye Tian, Department of Radiotherapy and Oncology, The Second Affiliated Hospital of Soochow University, 1055 Sanxiang Road, Suzhou, Jiangsu 215000, P.R. China

E-mail: dryetian@126.com

Key words: Kruppel-like factor 8, lung adenocarcinoma, Ki67, prognosis treat lung cancer, the 5-year survival rate of lung cancer is only $10-15 \%(2,3)$.

Kruppel-like factor 8 (KLF8) is a member of the KLF family and has various roles in the regulation of the cell cycle, apoptosis, proliferation, differentiation, development and carcinogenesis (4-6). Previous studies have demonstrated that KLF8 is identified in various types of cancer to a large extent, including gastric, lung, ovarian, breast and renal cancer (7-9). KLF8 may also affect the invasion and metastasis of tumors (10). However, no data is currently available on KLF8 in lung adenocarcinoma (LAC).

$\mathrm{Ki67}$ is a nuclear protein that is associated with cellular proliferation (11). Ki67 protein is present during all active phases of the cell cycle (G1, S, G2 and mitosis) (11). Ki67 has a short life span, thus it is absent in resting cell phases (G0) (12). $\mathrm{Ki67}$ has been widely used as a cell proliferation marker to determine the degree of growth, invasion and prognosis of cancer (13).

The present study aimed to investigate the expression of KLF8 in LAC, the association between KLF8 and clinical features, and the expression of Ki67 in patients with LAC. The relationship between KLF8 and patient survival was also investigated in Kaplan-Meier survival curves.

\section{Materials and methods}

LAC tissues were collected from 140 patients who underwent surgical resection without preoperative systemic chemotherapy or radiotherapy at the Affiliated Hospital of Nantong University (Nantong, China) between January 2009 and December 2010. LAC tissues were obtained by surgery using protocols approved by the Ethics Committee of the Affiliated Hospital of Nantong University. Written informed consent was provided by all patients enrolled in the study. Among the cases, there were 72 male and 68 female patients. These patients were aged between $39-77$ years, and the mean age was 61 years. All samples were fixed in $10 \%$ buffered formalin for $24 \mathrm{~h}$, which was performed at $20^{\circ} \mathrm{C}$ and embedded in paraffin at the time of collection. All 140 patients with LAC had corresponding history data and follow-up records.

Tumor tissues of these 140 specimens and corresponding tumor-adjacent tissues were used for construction of tissue 
microarrays (TMA). Briefly, each patient's tumor was represented by $2.0-\mathrm{mm}$ cores. Hematoxylin and eosin-stained slides (4- $\mu \mathrm{m}$ thick) for each patient were histologically analysed using an Olympus BX41 microscope (magnification, x200; Olympus Corporation, Tokyo, Japan) according to the the Union for International Cancer Control (UICC) TNM staging system predominantly for the scope of the primary tumor, regional lymph node metastasis and distant metastasis stage.

Another 8 samples of tumor tissues and adjacent non-tumor tissue were collected for western blot analysis. These samples were collected from 8 cases of patients who underwent curative resection between January 2009 and December 2010 of LAC tissues in the same hospital. Fresh samples were frozen in liquid nitrogen immediately after surgical removal and maintained at $-80^{\circ} \mathrm{C}$ until they were used for western blot analysis.

Western blot analysis. Protein was extracted from $\sim 0.1 \mathrm{~g}$ of fresh tissue from the 8 cases of tumor and matched adjacent normal samples. Tissues were immediately homogenized in a homogenization buffer containing $50 \mathrm{mM}$ Tris- $\mathrm{HCl}$ (pH 7.5), $150 \mathrm{mM} \mathrm{NaCl}, 0.1 \% \mathrm{NP}-40,5 \mathrm{mM}$ EDTA, $60 \mathrm{mM}$ $\beta$-glycerophosphate, $0.1 \mathrm{mM}$ sodium orthovanadate, $0.1 \mathrm{mM}$ $\mathrm{NaF}$ and complete protease inhibitor cocktail (Roche Diagnostics, Basel, Switzerland), and then centrifuged at $12,000 \mathrm{x} \mathrm{g}$ for $30 \mathrm{~min}$ to collect the supernatant $\left(4^{\circ} \mathrm{C}\right)$. The bicinchoninic acid assay method was used to determine the protein concentration in the samples after the addition of SDS buffer and $100^{\circ} \mathrm{C}$ for $10 \mathrm{~min}$. Following this, protein samples $(60 \mu \mathrm{g})$ were separated with $12 \%$ SDS-PAGE and transferred to polyvinylidene fluoride membranes. The membranes were blocked with Tris-buffered saline with Tween-20 (TBST) supplemented with 5\% skimmed milk for $1 \mathrm{~h}$ and then incubated with rabbit anti-human KLF8 antibody (1:150; ARP31533 P050; Aviva Systems Biology Corp., San Diego, CA, USA) or mouse anti-human GAPDH antibody (1:150; OAAD00231; Aviva Systems Biology Corp) overnight at $4^{\circ} \mathrm{C}$. Membranes were washed three times with TBST and incubated with goat anti-rabbit IgG-HRP $(1: 8,000 ;$ sc-2004) and goat anti-mouse IgG-HRP (1:5,000; sc-2005; both from Santa Cruz Biotechnology, Inc., Santa Cruz, USA) secondary antibodies for $2 \mathrm{~h}$ at room temperature. Enhanced chemiluminescence reagents (Thermo Fisher Scientific, Inc.) were used for band detection using a Tanon 5200 Imaging System (Tanon Science Technology Co., Shanghai, China). The band intensity was measured using the Image 1.40 analysis system (National Institutes of Health, Bethesda, MD, USA).

Immunohistochemistry. The EnVision method (14) was used for immunohistochemical staining. A total of 140 LAC samples were produced for TMA. The samples were incubated for $40 \mathrm{~min}$ at $70^{\circ} \mathrm{C}$, dewaxed and dehydrated in graded ethanol. Using TMA in antigen repairing $22 \mathrm{~min}$ at $99^{\circ} \mathrm{C}$, dripping with $3 \%$ hydrogen peroxide for $20 \mathrm{~min}$ at room temperature. Then the samples were washed with phosphate-buffered saline with Triton X-100 three times, and incubated with rabbit anti-human KLF8 antibody (1:150; Aviva Systems Biology Corp.) or rabbit anti-human Ki67 (1:500; ab15580; Abcam, Cambridge, UK) overnight at $4^{\circ} \mathrm{C}$. Subsequently, the samples were incubated with goat anti-rabbit IgG-HRP antibody (1:500; sc-2004; Santa Cruz Biotechnology, Inc.) for $2 \mathrm{~h}$ at room temperature.

\section{$\frac{1}{T N} \frac{2}{T N} \frac{3}{T N} \frac{4}{T N} \frac{5}{T N} \frac{6}{T N} \frac{7}{T N} \frac{8}{T N}$}

KLF8

GAPDH

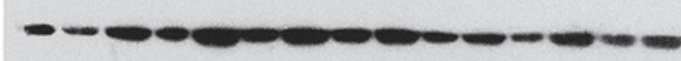

Figure 1. Expression of KLF8 was detected in 8 paired cases of lung adenocarcinoma and adjacent non-tumor tissue. GAPDH was used as a loading control. T, tumor tissue; N, non-tumor tissue; KLF8, Kruppel-like factor 8.

PBS was used as a negative control instead of the primary antibody. The Olympus BX41microscope was used to capture images of the samples.

Evaluation of staining. The analysis of these sections following immunostaining was conducted by two independent experienced pathologists. Both pathologists were unaware of the clinical pathological data and patient's outcome. KLF8 was predominantly located in the cytomembrane, also it was also observed in the cytoplasm and nuclei. Ki67 was also located in tumor cell nuclei. The positive cells were those with yellow/brown granules in the nucleus and whose staining intensity was higher than those with specific staining in the background. Under the microscope (magnification, x400), 100 cells were counted at each site, and the staining intensity and the percentage of positive cells were recorded in four sites. The staining intensity score assessment was as follows: 0 , no staining; 1 , weakly stained; 2 , moderately stained; and 3 , strongly stained. The final score was the product of KLF8 and Ki67 staining: $<3$ points was defined as negative and low staining; and $\geq 3$ points was defined as positive and high staining.

Statistical analysis. Statistical analysis was performed using SPSS v. 21.0 (IBM Corp., Armonk, NY, USA). The relationship between KLF8, Ki67 and clinical pathological features of lung adenocarcinoma were examined using $\chi^{2}$ testing. The correlation between KLF8 and Ki67 was analyzed according to Spearman's rank correlation. Survival curves were calculated by the Kaplan-Meier method. $\mathrm{P}<0.05$ was considered to indicate a statistically significant difference.

\section{Results}

KLF8 is present in LAC samples at high levels. In an attempt to verify whether KLF8 was involved in LAC, protein expression levels of KLF8 in 8 tumor tissue samples and adjacent non-tumor tissue were analyzed by western blot analysis. It was demonstrated that KLF8 expression levels were markedly higher in tumor samples than in normal lung samples (Fig. 1). Immunohistochemical staining was conducted on 140 LAC samples. As demonstrated in Fig. 2, positive cell percentages of KLF8 were as follows: $<10 \%$ (Fig. 2E); F, 11-50\% (Fig. 2F); G, $51-75 \%$ (Fig. 2G); and $>75 \%$ (Fig. 2H).

Correlation exists between KLF8 expression and clinical pathological parameters in patients with LAC. The relationship between KLF8 protein expression levels and patient 
Table I. KFL8 and Ki67 expression and clinicopathological parameters in 140 lung adenocarcinoma samples.

\begin{tabular}{|c|c|c|c|c|c|c|}
\hline \multirow[b]{2}{*}{ Parameters } & \multirow[b]{2}{*}{ Total } & \multicolumn{2}{|c|}{ KLF8 expression } & \multirow[b]{2}{*}{$\%$ of total } & \multirow[b]{2}{*}{$\chi^{2}$} & \multirow[b]{2}{*}{ P-value } \\
\hline & & Low & High & & & \\
\hline Sex & & & & & & 0.057 \\
\hline Male & 72 & 34 & 38 & 52.8 & 3.623 & \\
\hline Female & 68 & 43 & 25 & 36.8 & & \\
\hline Age, years & & & & & & 0.731 \\
\hline$<60$ & 60 & 34 & 26 & 43.3 & 0.118 & \\
\hline$\geq 60$ & 80 & 43 & 37 & 46.3 & & \\
\hline Tumor size, $\mathrm{cm}$ & & & & & & 0.170 \\
\hline$<3$ & 60 & 37 & 23 & 38.3 & 1.886 & \\
\hline$\geq 3$ & 80 & 40 & 40 & 50.0 & & \\
\hline Differentiation & & & & & & 0.035 \\
\hline Well & 33 & 23 & 10 & 30.3 & 6.707 & \\
\hline Moderate & 71 & 40 & 31 & 43.7 & & \\
\hline Poor & 36 & 14 & 22 & 61.1 & & \\
\hline TNM stage & & & & & & 0.001 \\
\hline I & 69 & 48 & 21 & 30.4 & 16.915 & \\
\hline II & 34 & 17 & 17 & 50.0 & & \\
\hline III & 31 & 12 & 19 & 61.3 & & \\
\hline IV & 6 & 0 & 6 & 100 & & \\
\hline Lymph node metastasis & & & & & & 0.015 \\
\hline No & 78 & 50 & 28 & 35.9 & 5.896 & \\
\hline Yes & 62 & 27 & 35 & 56.5 & & \\
\hline Median survival, months & & & & & & 0.018 \\
\hline$<37$ & 69 & 31 & 38 & 55.1 & 5.577 & \\
\hline$\geq 37$ & 71 & 46 & 25 & 35.2 & & \\
\hline Ki67 expression & & & & & & $<0.01$ \\
\hline High & 101 & 43 & 58 & 57.4 & 22.618 & \\
\hline Low & 39 & 34 & 5 & 12.8 & & \\
\hline
\end{tabular}

KLF8, Kruppel-like factor 8 .

clinical pathological parameters (Table I) was analyzed. KLF8 expression was significantly correlated with differentiation $(\mathrm{P}=0.035)$, TNM $(\mathrm{P}=0.001)$, lymph node metastasis $(\mathrm{P}=0.015)$ and median survival $(\mathrm{P}=0.018)$. However, no significant association was identified between KLF8 expression and the other clinical pathological characteristics, including sex, age and tumor size. Furthermore, there was a significant positive association between KLF8 and Ki67 expression (Table II; $\mathrm{P}<0.01$ ). Additionally, the correlation between KLF8 and Ki67 was analyzed by using Pearson's rank correlation, which indicated a significant positive correlation between the expression of these proteins ( $\mathrm{r}=0.511$; $\mathrm{P}<0.05$; Fig. 3 ).

KLF8 and Ki67 expression correlates with patient survival. The association between KLF8 expression and patient survival status was investigated. Kaplan-Meier survival curves demonstrated that high expression of KLF8 was significantly correlated with poor overall patient survival (Fig. 4A; $\mathrm{P}=0.011$ ). The prognosis of patients with high expression of KLF8 was
Table II. Correlation between expression of KLF8 and Ki67 in 140 lung adenocarcinoma specimens.

\begin{tabular}{lccccc}
\hline & \multicolumn{2}{c}{$\begin{array}{c}\text { KLF8 } \\
\text { expression }\end{array}$} & & \\
\cline { 2 - 4 } Ki67 expression & High & Low & r & P-value \\
\hline High & 58 & 43 & 0.402 & $<0.01$ \\
Low & 5 & 34 & & \\
\hline
\end{tabular}

KLF8, Kruppel-like factor 8.

worse than the prognosis of patients with lower expression levels. Furthermore, patients with higher expression levels of Ki67 demonstrated a significantly reduced survival rate compared with those who had low expression levels of Ki67 $(\mathrm{P}<0.01$; Fig. 4B). 

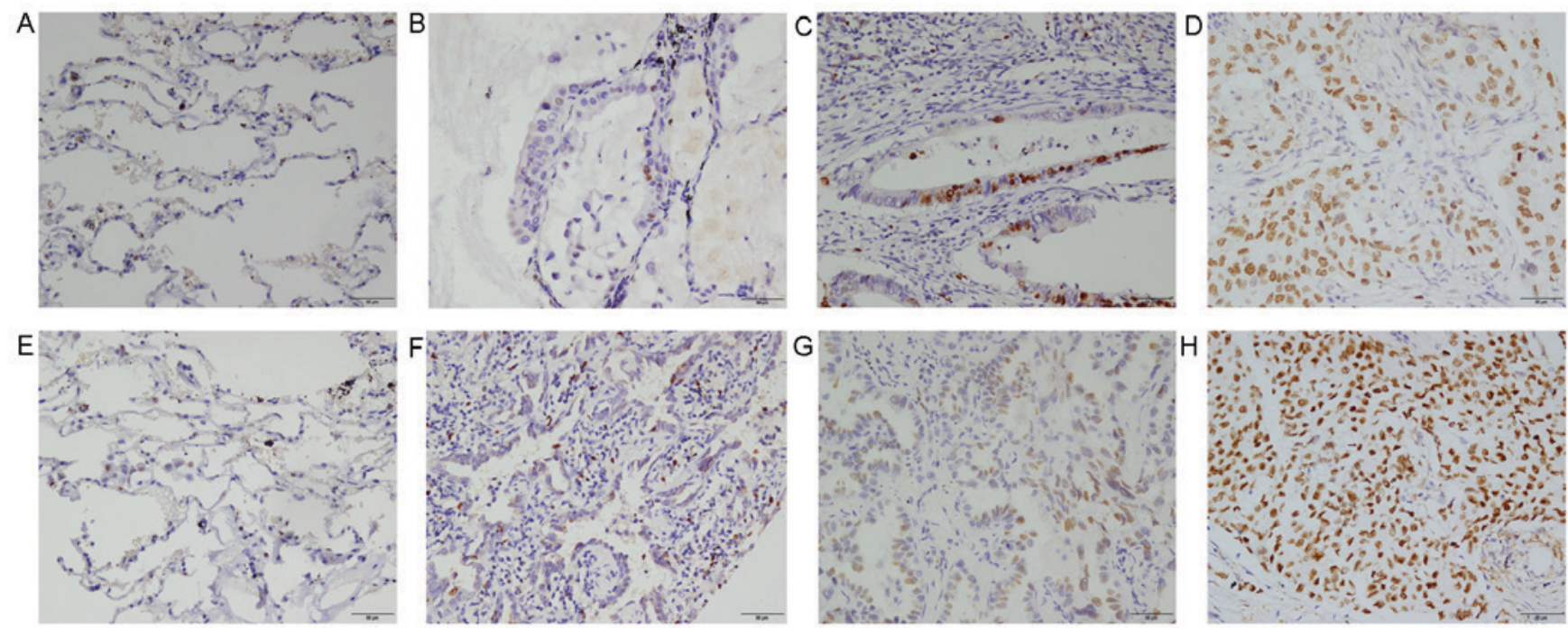

Figure 2. Expression of (A-D) Ki67 and (E-H) KLF8 in lung adenocarcinoma and adjacent non-tumor tissue by immunohistochemistry. (A and E) Adjacent non-tumor tissue. (B and F) Well-differentiated LAC. (C and G) Moderately differentiated LAC. (D and H) Poorly differentiated LAC. Magnification, x200; Scale bar, $50 \mu \mathrm{m}$. KLF8, Kruppel-like factor 8, LAC, lung adenocarcinoma.

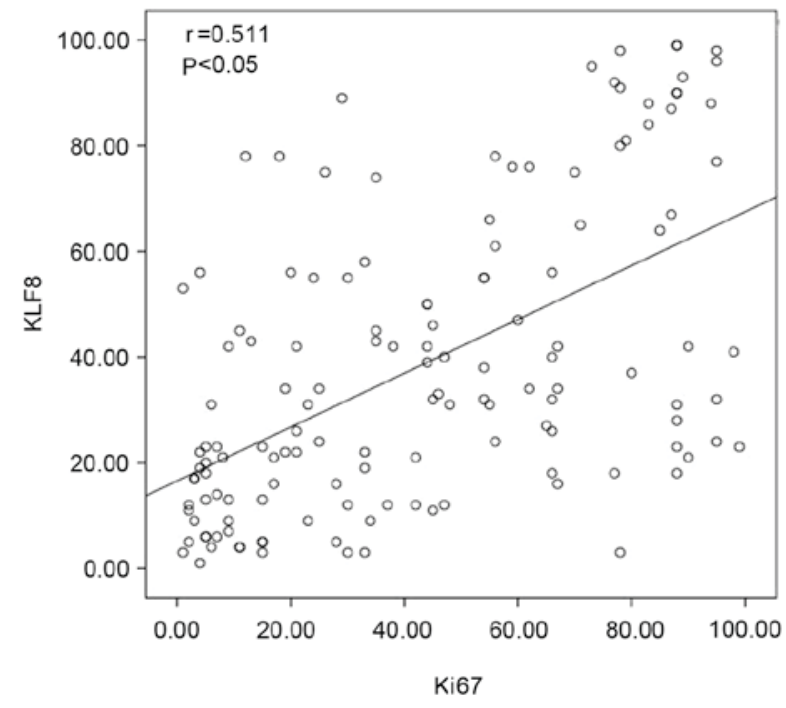

Figure 3. Correlation between KLF8 and Ki67 expression in lung adenocarcinoma was further evaluated by Pearson's rank correlation. KLF8, Kruppel-like factor 8.

\section{Discussion}

Lung cancer is one of the most common malignant tumors in China (15). The main causes of lung cancer include smoking, environmental pollution, population aging and lifestyle changes (15). A variety of molecular pathways and proteins participate in the development of LAC, and the current medical level is incapable of diagnosing LAC at an early stage, meaning that it usually metastasizes before being clinically diagnosed (3). Therefore, it is vital to identify new specific molecules to predict LAC prognosis.

KLFs are recognized as transcriptional inhibitor proteins of the Kruppel-like cys2/His2 zinc finger protein family (16). KLFs are DNA-binding transcription regulation factors, with a highly conserved zinc finger structure in the C-terminal domain, which may combine with a variety of gene promoter
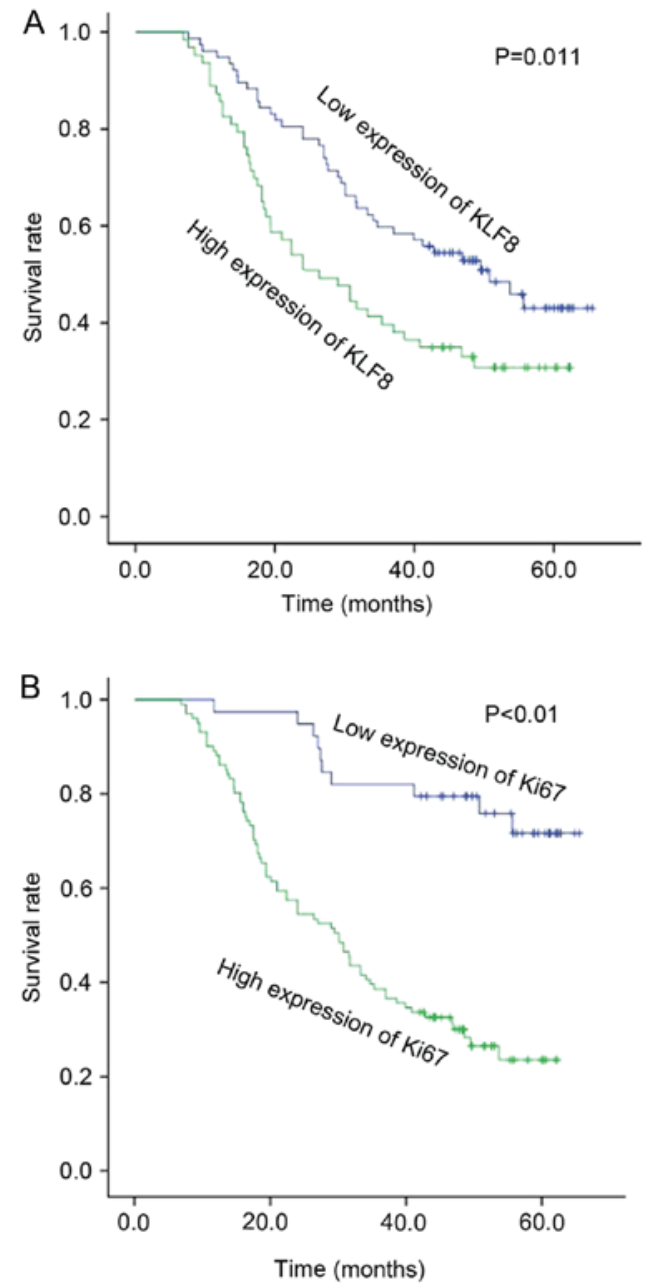

Figure 4. Overall survival curve of patients with lung adenocarcinoma according to high or low expression of (A) KLF8 and (B) Ki67. KLF8, Kruppel-like factor 8 .

components, particularly the GC box (17). The KLF family has an important role in cell cycle regulation and cell proliferation 
and also has an important function in the process of tumor development, invasion and metastasis $(4,18)$. The KLF family is composed of 14 members, KLF1-14. KLF8 was isolated from K562 leukemia and has been the focus of research (19). KLF8 is located on the X chromosome in humans and consists of 359 amino acid residues (20). The zinc fingers of KLF8 identify CACCC elements in DNA, and the full-length KLF8 may inhibit a CACCC-dependent promoter (21). Sometimes the zinc finger domain regulates protein-protein interactions $(4,16)$. Some researchers believe that KLF8 may be stimulated by small ubiquitin like modifiers (SUMO)-1, SUMO-2 and SUMO-3, primarily by SUMO-1 (17). Research has demonstrated that the function of KLF8 is limited when SUMO-1 is bound at Lys67 $(17,22,23)$.

Various studies have demonstrated that high expression of KLF8 exists in a variety of tumors, including as breast $(24,25)$, ovarian (26), bladder (27) and gastric cancer $(28,29)$. A previous study indicated that KLF8 may act on matrix metalloproteinase 9 transcription in breast cancer, promoting cell invasion and metastasis of human breast cancer (24). KLF8 is also tightly regulated by focal adhesion kinase through Src and phosphoinositide 3-kinase signaling pathways via transcription factors specificity protein 1, KLF1 and KLF3 (26,30). KLF8 also regulates the expression of vimentin and $\mathrm{N}$-cadherin, and increases the expression of $\beta$-catenin, thus promoting the proliferation and migration of bladder cancer cells (27). In addition, KLF8 regulates epithelial-mesenchymal transition (EMT) induced by transforming growth factor (TGF)- $\beta 1$ in gastric cancer cells (28). Downregulation of KLF8 by small interfering RNA has been demonstrated to block TGF- $\beta 1$-induced EMT-like transformation and TGF- $\beta 1$-prompted cell migration, invasion and motility (28).

In the present study, the expression of KLF8 in 8 paired cases of fresh lung adenocarcinoma tissues and non-cancerous tissues was investigated using western blotting. The results demonstrated that the expression of KLF8 in lung adenocarcinoma was markedly higher than that in non-cancerous tissues. Additionally, the expression of KLF8 and Ki67 in patients with LAC using TMA was evaluated. High expression of KLF8 was significantly correlated with differentiation, TNM and lymph node metastasis. Furthermore, the expression of KLF8 was correlated positively with Ki67. The patients with high expression levels of KLF8 or Ki67 had a worse prognosis than those with low expression levels of KLF8. In conclusion, the KLF8 protein may be a novel therapeutic target for LAC; however, further study of the molecular mechanisms of KLF8 in $\mathrm{LAC}$ is required.

\section{Acknowledgements}

The present study was supported by the Suzhou Science and Technology Development Program (grant no. SZS201509), the Jiangsu Provincial Special Program of Clinical Medical Science (grant no. BL2014040).

\section{References}

1. Liu Y, Lv L, Xue Q, Wan C, Ni T, Chen B, Liu Y, Zhou Y, Ni R and Mao G: Vacuolar protein sorting 4B, an ATPase protein positively regulates the progression of NSCLC via promoting cell division. Mol Cell Biochem 381: 163-171, 2013.
2. Rivera C,Pecuchet N,Wermert D,PricopiC,LePimpec-Barthes F, Riquet $\mathrm{M}$ and Fabre E: Obesity and lung cancer: Incidence and repercussions on epidemiology, pathology and treatments. Rev Pneumol Clin 71: 37-43, 2015 (In French).

3. Xu MM, Mao GX, Liu J, Li JC, Huang H, Liu YF and Liu JH: Low expression of the FoxO4 gene may contribute to the phenomenon of EMT in non-small cell lung cancer. Asian Pac J Cancer Prev 15: 4013-4018, 2014.

4. Evans PM and Liu C: New insights into KLF8-mediated transactivation. Cell Cycle 9: 649-650, 2010.

5. Shi H, Ji Y, Zhang D, Liu Y and Fang P: MiR-135a inhibits migration and invasion and regulates EMT-related marker genes by targeting KLF8 in lung cancer cells. Biochem Biophys Res Commun 465: 125-130, 2015.

6. Schnell O, Romagna A, Jaehnert I, Albrecht V, Eigenbrod S, Juerchott K, Kretzschmar H, Tonn JC and Schichor C: Krüppellike factor 8 (KLF8) is expressed in gliomas of different WHO grades and is essential for tumor cell proliferation. PLoS One 7: e30429, 2012.

7. Chen G, Yang W, Jin W, Wang Y, Tao C and Yu Z: Lentivirusmediated gene silencing of KLF8 reduced the proliferation and invasion of gastric cancer cells. Mol Biol Rep 39: 9809-9815, 2012.

8. Wang X, Urvalek AM, Liu J and Zhao J: Activation of KLF8 transcription by focal adhesion kinase in human ovarian epithelial and cancer cells. J Biol Chem 283: 13934-13942, 2008.

9. Fu WJ, Li JC, Wu XY, Yang ZB, Mo ZN, Huang JW, Xia GW, Ding Q, Liu KD and Zhu HG: Small interference RNA targeting Krüppel-like factor 8 inhibits the renal carcinoma 786-0 cells growth in vitro and in vivo. J Cancer Res Clin Oncol 136: $1255-1265,2010$.

10. Wang $X$ and Zhao J: KLF8 transcription factor participates in oncogenic transformation. Oncogene 26: 456-461, 2007.

11. Schwab U, Stein H, Gerdes J, Lemke H, Kirchner H, Schaadt M and Diehl V: Production of a monoclonal antibody specific for Hodgkin and Sternberg-Reed cells of Hodgkin's disease and a subset of normal lymphoid cells. Nature 299: 65-67, 1982.

12. Leuverink EM, Brennan BA, Crook ML, Doherty DA, Hammond IG, Ruba S and Stewart CJ: Prognostic value of mitotic counts and Ki-67 immunoreactivity in adult-type granulosa cell tumour of the ovary. J Clin Pathol 61: 914-919, 2008.

13. Melling N, Kowitz CM, Simon R, Bokemeyer C, Terracciano L, Sauter G, Izbicki JR and Marx AH: High Ki67 expression is an independent good prognostic marker in colorectal cancer. J Clin Pathol 69: 209-214, 2016.

14. Sabattini E, Bisgaard K, Ascani S, Poggi S, Piccioli M, Ceccarelli C, Pieri F, Fraternali-Orcioni G and Pileri SA: The EnVision++ system: A new immunohistochemical method for diagnostics and research. Critical comparison with the APAAP, ChemMate, CSA, LABC, and SABC techniques. J Clin Pathol 51: 506-511, 1998.

15. Siegel R, Ma J, Zou Z and Jemal A: Cancer statistics, 2014. CA Cancer J Clin 64: 9-29, 2014.

16. Rodriguez E and Martignetti JA: The Kruppel traffic report: Cooperative signals direct KLF8 nuclear transport. Cell Res 19: 1041-1043, 2009.

17. Lahiri SK and Zhao J: Krüppel-like factor 8 emerges as an important regulator of cancer. Am J Transl Res 4: 357-363, 2012.

18. Eaton SA, Funnell AP, Sue N, Nicholas H, Pearson RC and Crossley M: A network of Krüppel-like Factors (Klfs). Klf8 is repressed by Klf3 and activated by Klf1 in vivo. J Biol Chem 283: 26937-26947, 2008.

19. Lossi AM, Laugier-Anfossi F, Depetris D, Gecz J, Gedeon A, Kooy F, Schwartz C, Mattei MG, Croquette MF and Villard L: Abnormal expression of the KLF8 (ZNF741) gene in a female patient with an X;autosome translocation $\mathrm{t}(\mathrm{X} ; 21)(\mathrm{p} 11.2 ; \mathrm{q} 22.3)$ and non-syndromic mental retardation. J Med Genet 39: 113-117, 2002.

20. Lee H, Kim HJ, Lee YJ, Lee MY, Choi H, Lee H and Kim JW: Krüppel-like factor KLF8 plays a critical role in adipocyte differentiation. PLoS One 7: e52474, 2012.

21. van Vliet J, Turner J and Crossley M: Human Krüppel-like factor 8: A CACCC-box binding protein that associates with $\mathrm{CtBP}$ and represses transcription. Nucleic Acids Res 28: 1955-1962, 2000

22. Wei H, Wang X, Gan B, Urvalek AM, Melkoumian ZK, Guan JL and Zhao J: Sumoylation delimits KLF8 transcriptional activity associated with the cell cycle regulation. J Biol Chem 281: 16664-16671, 2006. 
23. Urvalek AM, Lu H, Wang X, Li T, Yu L, Zhu J, Lin Q and Zhao J: Regulation of the oncoprotein KLF8 by a switch between acetylation and sumoylation. Am J Transl Res 3: 121-132, 2011.

24. Wang X, Lu H, Urvalek AM, Li T, Yu L, Lamar J, DiPersio CM Feustel PJ and Zhao J: KLF8 promotes human breast cancer cell invasion and metastasis by transcriptional activation of MMP9. Oncogene 30: 1901-1911, 2011.

25. Lu H, Hu L, Yu L, Wang X, Urvalek AM, Li T, Shen C, Mukherjee D, Lahiri SK, Wason MS and Zhao J: KLF8 and FAK cooperatively enrich the active MMP14 on the cell surface required for the metastatic progression of breast cancer. Oncogene 33: 2909-2917, 2014.

26. Lu H, Wang X, Urvalek AM, Li T, Xie H, Yu L and Zhao J: Transformation of human ovarian surface epithelial cells by Krüppel-like factor 8. Oncogene 33: 10-18, 2014.
27. Liang K, Liu T, Chu N, Kang J, Zhang R, Yu Y, Li D and Lu D: KLF8 is required for bladder cancer cell proliferation and migration. Biotechnol Appl Biochem 62: 628-633, 2015.

28. Zhang H, Liu L, Wang Y, Zhao G, Xie R, Liu C, Xiao X, Wu K, Nie Y, Zhang H and Fan D: KLF8 involves in TGF-beta-induced EMT and promotes invasion and migration in gastric cancer cells. J Cancer Res Clin Oncol 139: 1033-1042, 2013.

29. Hsu LS, Wu PR, Yeh KT, Yeh CM, Shen KH, Chen CJ and Soon MS: Positive nuclear expression of KLF8 might be correlated with shorter survival in gastric adenocarcinoma. Ann Diagn Pathol 18: 74-77, 2014.

30. Zhao J, Bian ZC, Yee K, Chen BP, Chien S and Guan JL: Identification of transcription factor KLF8 as a downstream target of focal adhesion kinase in its regulation of cyclin D1 and cell cycle progression. Mol Cell 11: 1503-1515, 2003. 